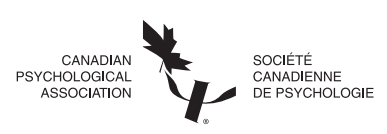

\title{
Interactive Rhythms in the Wild, in the Brain, and in Silico
}

\author{
Marianna Anichini \\ Hanse-Wissenschaftskolleg (Institute for Advanced Study) and \\ Carl von Ossietzky University Oldenburg
}

\author{
Marianne de Heer Kloots \\ University of Amsterdam
}

\author{
Andrea Ravignani \\ Max Planck Institute for Psycholinguistics and Sealcentre Pieterburen, Pieterburen, the Netherlands
}

\begin{abstract}
There are some historical divisions in methods, rationales, and purposes between studies on comparative cognition and behavioural ecology. In turn, the interaction between these two branches and studies from mathematics, computation, and neuroscience is not usual. In this short piece, we attempt to build bridges among these disciplines. We present a series of interconnected vignettes meant to illustrate what a more interdisciplinary approach looks like when successful, and its advantages. Concretely, we focus on a recent topic, namely animal rhythms in interaction, studied under different approaches. We showcase 5 research efforts, which we believe successfully link 5 particular scientific areas of rhythm research conceptualised as the following: social neuroscience, detailed rhythmic quantification, ontogeny, computational approaches, and spontaneous interactions. Our suggestions will hopefully spur a "comparative rhythms in interaction" field, which can integrate and capitalize on knowledge from zoology, comparative psychology, neuroscience, and computation.
\end{abstract}

\section{Public Significance Statement}

Why do we see so many rhythmic processes interacting in human behaviour, in other animals, and when studying brains? Are they all connected? In this article, we highlight some fruitful links among research disciplines all focusing on interactive rhythms but from different traditions, spanning social neuroscience, computational modelling, animal behaviour and cognitive development.

Keywords: communicative rhythms in behaviour, brain rhythms, comparative cognition, rhythm ontogeny, agent-based models

The last decade has seen an explosion of comparative research on the cognitive and communicative roots of animal rhythmic behaviour. Emphasis has been laid on rhythms in vocal communication and motoric rhythms in response to sound.

There is a clear tendency to increase interdisciplinary research, but still sometimes current work in, for example, behavioural ecology or cognitive neuroscience forgets related studies from the comparative cognition tradition, and vice versa. In particular, the comparative study of interactive rhythms is an area gaining increasing attention. As it happens for most new research topics, it suffers from a disconnection among several methodological approaches and theoretical traditions. Of course. exceptions exist, such as the legendary biologist William Hamilton, who enjoyed and performed mathematical modelling of biological processes as much as pure fieldwork (Herbers, 2013).

In this opinion piece, we focus on interactive rhythms, particularly in the vocal domain: how individuals adjust the timing of their communicative acts in an interactive, social context. We aim first to show how interactive vocal rhythm research is rich and diverse, but still relatively sparse; second, to focus on key concepts
Marianna Anichini, BRAIN (Neurosciences and Cognitive Sciences), Hanse-Wissenschaftskolleg (Institute for Advanced Study), and Division Animal Physiology and Behavior, Department for Neuroscience, School of Medicine and Health Sciences and Cluster of Excellence Hearing4all, Carl von Ossietzky University Oldenburg; (D) Marianne de Heer Kloots, Institute for Advanced Study, University of Amsterdam; (D) Andrea Ravignani, Comparative Bioacoustics Group, Max Planck Institute for Psycholinguistics, and Research Department, Sealcentre Pieterburen, Pieterburen, the Netherlands.

Marianna Anichini and Marianne de Heer Kloots share joint first authorship.
Marianna Anichini was supported via the Hanse-Wissenschaftskolleg (Institute for Advanced Study) with the Junior Fellowship. Andrea Ravignani was supported via the European Union Horizon, 2020 research and innovation programme Marie Skłodowska-Curie grant, agreement 665501 with the research Foundation Flanders (FWO) (Pegasus2 Marie Curie fellowship $12 \mathrm{~N} 5517 \mathrm{~N})$.

Correspondence concerning this article should be addressed to Andrea Ravignani, Comparative Bioacoustics Group, Max Planck Institute for Psycholinguistics, Wundtlaan 1,6525 XD Nijmegen, the Netherlands. Email: andrea.ravignani@mpi.nl 
from a few papers, to highlight fruitful cases of connection between strands; and third to imply how, at least in this subarea, comparative cognition and behavioural ecology can smoothly blend into each other.

To achieve these aims, we discuss and try to connect five scientific areas, focusing on the following: social neuroscience, with emphasis on how social context affects brain rhythms, and how different functional areas of the brain are connected, comparing connectivity patterns across species; detailed rhythmic quantification, used to detect regularities in rhythm and to compare these across species; ontogeny of vocal rhythms, based on speech development work; computational approaches, using agent-based modelling to compare animal behaviours with those simulated by a computer model under a set of known assumptions; and spontaneous interactions in ecological contexts, relating environmental stimuli, sensory biases, and underlying brain mechanisms.

Our goal is to sketch the interdisciplinary synergy among these five scientific areas, by focusing on five research efforts. Each of these efforts connects two of the areas above, hence illustrating a potential practical link (see Figure 1). We start with two papers, by Lindenberger, Li, Gruber, and Müller (2009) and Dikker and colleagues (2017), studying spontaneous interactions within the context of human social neuroscience, investigating the brain rhythms underlying socioecological interactions. This research effort is included in the first section, titled "Humans: Social Neuroscience of Spontaneous Interactions". We then move to the research effort of Okobi, Banerjee, Matheson, Phelps, and Long (2019), titled "Mice: Detailed Quantification of Social Rhythms." This research probes the neural underpinnings of natural vocal rhythms in singing mice, and connects the social neuroscience and detailed rhythmic quantification areas. Afterward, the third effort is included in the section named "Birds: Measuring Rhythmic Ontogeny". In this section, we present works by Hyland Bruno (2017) and Hyland Bruno and Tchernichovski (2017) regarding the development and learning processes underlying rhythm in bird songs, which connects detailed rhythmic quantification with ontogeny. The fourth link, presented in the section "Agent-Based Models of Rhythm Development", is between ontogeny and agentbased modelling of interacting agents illustrated through a paper on rhythmic capacities of a seal pup (Ravignani, 2019). The last research effort is titled "Tree Frogs: Agent-Based Modelling of Spontaneous Interactions." Specifically, it describes the work done by Aihara et al. (2014). This last paper "closes the circle" (see Figure 1) connecting the agent-based modelling with the social spontaneous interactions scientific areas.

\section{Humans: Social Neuroscience of Spontaneous Interactions}

In research on cognitive functioning, in both human and nonhuman animals, neuroimaging studies have traditionally tested single individuals in highly controlled lab environments. However, calls for more interactive experimental settings (Schilbach et al., 2013) as well as more ecologically relevant behavioural tasks (Krakauer, Ghazanfar, Gomez-Marin, MacIver, \& Poeppel, 2017) are spurring these strands of research to analyse neural data through a more ethological lens. Although the structural neurobiology needed for vocal communication is under investigation in many species (Kelley \& Bass, 2010) and the neural activity underlying naturalistic social interactions has long been studied in some (e.g., Hopkins, 1988), cognitive neuroscience can still bring novel insights to the table on interpersonal neural synchronization in human temporal coordination and social interactions.

Electroencephalography (EEG) hyperscanning techniques, in which neural electric signals are recorded from multiple participants simultaneously, have been employed for studying the neuroscience of human social interactions (Babiloni \& Astolfi, 2014; Liu et al., 2018). Given their high temporal resolution, EEG

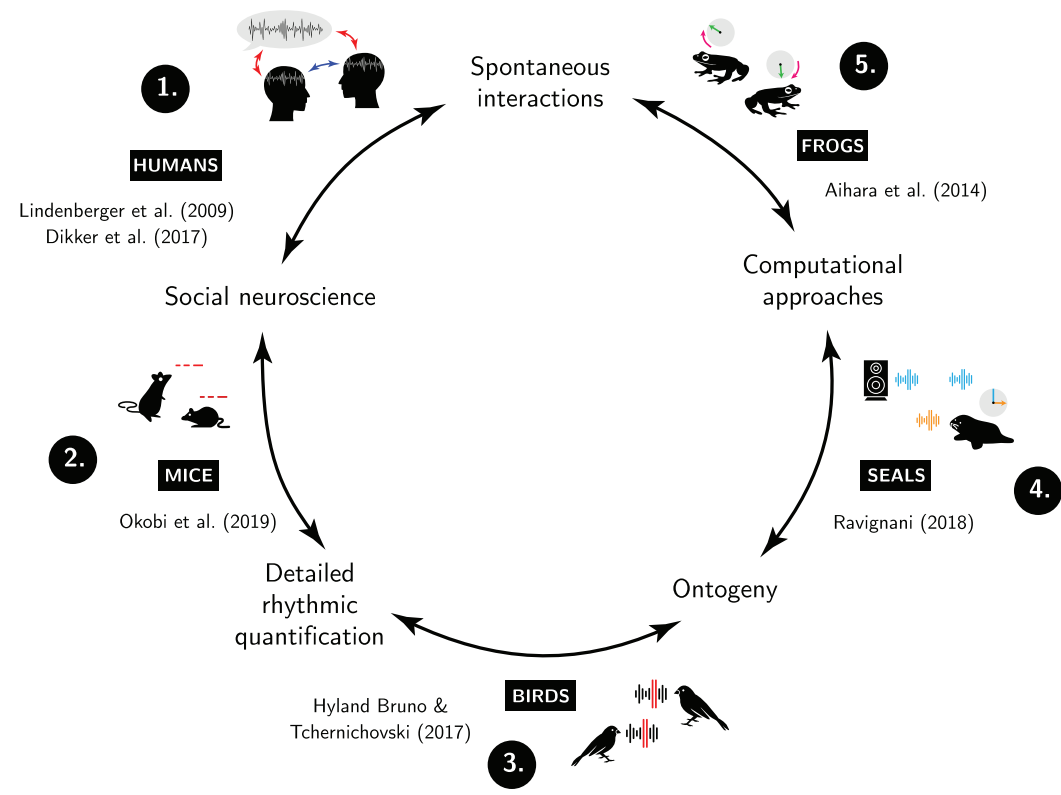

Figure 1. Pairwise links between two of the five scientific areas of interest via the key five research efforts and species discussed here. See the online article for the color version of this figure. 
hyperscanning measurements seem particularly suited for studying interactive rhythmicity.

In the musical domain, Lindenberger et al. (2009) used EEG hyperscanning to measure synchronized neural activity between pairs of participants playing the guitar. They found that phase synchronization increased significantly when preparing for and coordinating play onset, and argue that between-brain oscillatory couplings reflect between-participants similarities in the temporal properties of their percepts and actions.

Using low-cost portable EEG systems, hyperscanning techniques can also be brought to the "field," allowing testing for interbrain coupling in a broader number of situations. Dikker and colleagues (2017) investigated spontaneous neural synchronization to environmental input under radically naturalistic circumstances compared to usual neuroscientific approaches: They simultaneously recorded a group of high school students during regular class activity. Measuring brain-to-brain synchronization across the entire group, the authors found that the degree of neural synchronization among students could predict both social dynamics (group affinity and empathy) and class engagement (self-reported focus and teaching style appreciation). Dikker and colleagues (2017) argue that between-brain synchronization here is driven by shared attention mechanisms: Neural oscillations become more similar among students when they are paying attention, because they entrain to the same external stimulus (e.g., the teacher who is lecturing).

These studies highlight promising ways to investigate the neural underpinnings of human real-world musical and vocal interaction. Both studies demonstrate that interacting neural rhythms can be used as physiological markers of real-world social interaction on a behavioural level. Lindenberger and colleagues (2009) show that coordinated rhythmic behaviour is associated with interpersonal synchronization on the neural level, and Dikker and collaborators (2017) reveal that such synchronized neural rhythms also predict more complex human social dynamics in truly naturalistic settings.

Other work, on spontaneous brain-to-stimulus synchronization, indicates that this mechanism correlates both with neuroanatomical connectivity patterns and performance on ecologically relevant tasks like word learning (Assaneo et al., 2019).

Carefully designed studies like these showcase opportunities for the interpretation of human neural data in behaviourally relevant contexts. Taken together, these methods provide encouraging steps toward a better understanding of the brain mechanisms behind real-world human vocal rhythms.

\section{Mice: Detailed Quantification of Social Rhythms}

Social interactions, including human music making and conversation, involve the dynamic modulation of actions based on cues picked up in the behaviour of others. In the study of vocal rhythms, acoustic exchanges among conspecifics are ideal test cases to probe such sensorimotor transformations. Despite the ubiquity of timed acoustic interactions, well-described across taxa in behavioural ecology (Bee \& Micheyl, 2008; Pika, Wilkinson, Kendrick, \& Vernes, 2018; Ravignani, Verga, \& Greenfield, 2019; Schwartz \& Freeberg, 2008), few animal models have been examined neuroscientifically.

Okobi and colleagues (2019) introduced Alston's singing mice (Scotinomys teguina) as a new mammal model exhibiting turn- taking behaviour. To inspect the causal dynamics behind these precisely timed vocal interactions, they used an innovative combination of behavioural measures and neural techniques. Comparing isolated singing to countersinging in duets, Okobi and colleagues (2019) found that individual vocal productions were more stereotyped in isolation and showed more variation in duetting. Specific brain centres were mapped to jaw movements during song production by measuring muscle cell electricity (electromyography) and electrically stimulating neural populations (intracortical microstimulation), highlighting the orofacial motor cortex (OMC) as a crucial centre controlling song-related musculature. Subsequent perturbation and focal cooling experiments targeted at the OMC during isolated singing showed that disrupting the OMC alters song progression without influencing individual note structure. Finally, the causal role of the OMC in social vocalizations was corroborated in a playback experiment: Countersinging responses to playback songs were absent in mice where the OMC was pharmacologically deactivated, while they were successfully elicited in a control group.

Okobi and colleagues conclude that a hierarchical mechanism underlies countersinging in Alston's singing mice, with distinct functional regions controlling vocal production and coordination: The OMC influences the pacing of songs, without altering the structure of individual notes. The distinction between temporal structure on the level of the song and the level of individual notes is well characterised through the authors' measure of the "song trajectory slope": Plotting note duration against note onset time, it becomes obvious that these slopes for individual mice are much more variable in social than in isolated settings.

This study convincingly shows that, for Alston's singing mice, involvement of the motor cortex is necessary for successful vocal interaction, whereas previous mammalian models have assumed that subcortical structures alone are sufficient for vocal timing (see, e.g., Jürgens, 2009). The clever design of behavioural measures (e.g., the song trajectory slope) was essential for this finding: These detailed measures accurately captured differences in subcomponents of vocal rhythmicity among social contexts, which could then be causally linked to neural dynamics.

\section{Birds: Measuring Rhythmic Ontogeny}

In several vertebrates, precisely timed sequences of behaviour are not innate but are acquired by juvenile animals (Aronov, Veit, Goldberg, \& Fee, 2011). In songbirds, for instance, mature song emerges gradually from an unstructured immature subsong, until it matches the internal template of the adult song (Konishi, 1965). Still, little is known about how the rhythmic components of songs are learned, in order to answer questions like "How do song rhythms become consolidated?" and "How do different timescales of song rhythm relate to one another?"

Studies conducted by Hyland Bruno and Tchernichovski investigated song-rhythm learning in songbirds (Hyland Bruno, 2017; Hyland Bruno \& Tchernichovski, 2019). Zebra finches learn their songs by imitating an adult (Marler, 1970). Although mature songs exhibit hierarchical organisation, the repetitions of motifs-the bout-are no longer stereotyped. Indeed, in a first study (Hyland Bruno \& Tchernichovski, 2019), the authors found patterns of flexibility in the rhythmic organisation of song bouts, visualizing them as sorted raster plots of acoustic features. They showed that 
when motifs $(\mathrm{M})$ were strung together $(\mathrm{M}-\mathrm{M})$ with two or three intervening short calls, named connectors (c, leading to M-cc-M, M-ccc-M), the time interval between the motifs was highly variable, allowing a variation in timing across singing performances. Even among co-tutored birds that learned the same motif, the authors observed strong individual variability in the temporal plasticity of song bouts (Hyland Bruno \& Tchernichovski, 2019). During song development, the juvenile bird filters the influence of an external auditory model through a previously internalized rhythm template.

Controlled song-learning experiments were further performed in a second study (Hyland Bruno, 2017), which tested whether the song rhythm is learned, or is instead an epiphenomenon of the learning of temporal sequences. Initially, birds were trained to learn to imitate a regularly timed (isochronous), monosyllabic song sequence (AAAA). Afterward, when the birds learned the song with "AAAA" sequence, a new song target "ABAB" was introduced with an additional syllable. Birds were hence divided into two experimental groups. In the first one, the tested birds heard the new syllable B congruent with the existing rhythm: The song "AAAA" had the same isochronous rhythm of "ABAB." In the second group, they were tested on a new target non-isochronous song, where the new syllable was slightly shortened in duration. Hyland Bruno (2017) found that over ontogeny zebra finches more readily incorporated a new syllable when the tutored rhythm was unchanged, suggesting that a rhythmic pattern is established and used during song learning. An isochronous rhythm helped the birds adopt a new song sequence.

Based on these results, the authors propose an updated schema of the song imitation process, situating sequence learning within a rhythmic framework. The authors showed how juvenile birds develop their vocal learning capacity following the unstereotyped time structure of the adult's song bouts. These studies show that rhythmic coordination can be crucial for individuals engaging in vocal communicative acts, and highlight the importance of the social context and the rhythmic environment in which song learning develops.

\section{Seals: Agent-Based Models of Rhythm Development}

The ontogenesis of vocal rhythms is only sporadically investigated. This makes the predictions based on related empirical work quite vague, as the hypothesis space has not been constrained by previous experiments. In these particular cases, agent-based modelling can be useful, as it summarily probes the space of possible outcomes as a second-best option instead of relying on previous (in this case nonexistent) actual experiments.

An example of combining agent-based modelling with developmental rhythm research is provided by a recent case study in a harbour seal pup. A seal pup was probed for its capacity to vocally respond to simulated playbacks of conspecifics (Ravignani, 2019). As no similar experiment was run in this or related species, mechanisms observed in other taxa provided different hypotheses on the potential rhythmic behaviours of the seal pup.

Alternative, competing hypotheses on how the seal pup would react to rhythmic playbacks included 1) an arousal mechanism, by which an individual hearing more calls responds with more calls, with no rhythmic adaptation at all; the focal animal and the playback stimulus might have the same number of calls, which, however, do not have a systematic relation between their onsets; 2) adaptive synchrony, as often seen in human movement, by which an individual modifies the rate and delay of its behaviors so that they occur at the same time as those of a conspecific; 3) phase delay, as attested in bush crickets, which is based on a simple internal oscillator triggering sound production that gets reset every time a conspecific is heard; and 4) antisynchrony, as indirectly predicted by a classical theoretical model, by which an individual will modify the rate and delay of its calls so that they occur at a fraction of the period of a conspecific (Hamilton, 1971; Merker, Madison, \& Eckerdal, 2009; Ravignani, 2014; Ravignani \& de Reus, 2019).

Before running playback experiments, agent-based simulations were performed to see how four hypothetical seals, each adopting one of the four strategies above, would react to the playbacks. Data collected from the actual experiment were analysed with traditional statistical tools. In addition, however, comparison of the four models with the experimental data showed that the seal behaviour observed in the experiment matched one of the models (antisynchrony) and clearly differed from the others (Ravignani, 2019).

One additional angle, unfortunately not probed in this experiment, could be to use agent-based models to actually model developmental processes (e.g., de Boer, 2001; de Boer \& Kuhl, 2003). By collecting data over developmental stages, and running corresponding computer simulations, one could test how acoustic behaviours change over ontogeny while summarising their fundamental mechanisms.

\section{Tree Frogs: Agent-Based Modelling of Spontaneous Interactions}

Antisynchrony is also at the core of another paper, this time showing how two "strange bedfellows" can be fruitfully combined: work with animals in the field, and simulated computer agents acting according to theoretical models.

In particular, while studying choruses of Japanese tree frogs in the field, Aihara and colleagues (2014) built simple mathematical models of the frogs' vocal behaviour. The authors used a classic "phase oscillator model" (Ota, Aihara, \& Aoyagi, 2019; Strogatz, 2004). The gist of the model is that every agent (e.g., every frog) can be imagined as an analogue clock. Each clock has only one clock hand, potentially moving at a different speed from other clocks. Each clock's behaviour can be described by two main parameters: the angular velocity, namely how fast the hand runs (i.e., how fast time flows), and the phase, which is the direction the hand points to (i.e., the current time). If one hypothesizes that the two clocks can influence each other, the resulting "clock equations" for each agent can be noted mathematically and implemented in computer code. The power of this method is that all possible interactive scenarios among agents can be simulated, and the model can be modified and expanded with additional parameters.

To summarise the modelling side, the authors used simulations providing predictions on what an actual chorus in the field would look like (see also Aihara, 2009; Aihara et al., 2011). They then went to the field and measured the signalling behaviour of the actual frogs using a novel sound-imaging method, enabling realtime visualization of each frog call (Mizumoto et al., 2011). Analyses of the video recordings showed that two-cluster anti- 
synchronization was frequent in choruses of male Japanese tree frogs. The finding qualitatively confirms what the mathematical model previously predicted: the existence of two-cluster antisynchronization in the choruses of male Japanese tree frogs. However, the authors did not detect a wavy anti-synchronization in the field experiment, although it did appear as a stable solution in the numerical simulation (though less often observed than the twocluster anti-synchronization).

Aihara and colleagues (2014) motivated this inconsistency in terms of particular field condition occurring during the recordings. The sound images device was adopted only along one edge of the field where the larger numbers of male frogs aggregated. In such a setup, a straight-line distribution of the calling male frogs was approximately realised, compromising the wavy anti-synchronization of the male frogs' signalling behaviour.

Aihara and colleagues (2014) suggested that male Japanese tree frogs may dynamically change their calling times even in the same chorusing session beyond the expectation of the present mathematical model. Aiming to predict as many as possible spatiotemporal structures occurring within the collective animal behaviour dynamics should be the main task of mathematical models, predictions which can then be tested against empirical field research.

\section{Conclusions}

The aim of this short piece was not to perform an exhaustive literature review. Instead, we intended to link a few points at the intersection of science, scientific methodology, and metascience.

Apart from the overall connection among the few papers we have chosen, each of them individually contains important lessons on the added value of combining two or more approaches. The human studies show that valuable and ecologically valid insights on interpersonal cross-brain interactions can be obtained from recording EEG in social settings. The mice study shows how combining rhythm-tailored behavioural measurements with neurobiological techniques targeting specific areas can help establish fine-grained mechanisms for precise rhythmic behaviours. The bird studies show how long-term monitoring of all vocalizations produced by birds developing in controlled environments, combined again with advanced rhythm measurements, enables to track the long-term impacts of early social experiences and identify constraints on flexibility. The seal study first confirms the importance of studying the vocal behaviour in the early stage of the individual's life and, second, suggests how agent-based modelling can both provide predictions before an experiment is run, and confirm the empirical results after the experiment. The frog study shows how even spontaneous animal interactions in the wild can be mathematically modelled, hence combining ecological approaches with the precision of mathematics.

We realise that our usage of the term "rhythm," as in "vocal rhythms" and "interactive rhythms," is quite broad, and the behaviours we focus on could also be referred to as dynamic modulation of actions. Each field studying rhythm struggles with a clear definition (just for rhythm in music, for instance, see the many definitions in Toussaint, 2019). Our aim is to have a definition that, being as broad as possible, can be applied to virtually any animal behaviour or neural process.

To conclude, we are optimistic that many subareas at the boundary of comparative cognition and behavioural ecology will recip- rocally communicate and exchange theoretical and methodological approaches, as several others are already doing.

\section{Résumé}

Il existe des clivages historiques entre les méthodes, les justifications et les objectifs des études sur la cognition comparative et l'écologie comportementale. Par conséquent, les interactions entre ces deux branches et les études provenant des secteurs des mathématiques, de l'informatique et des neurosciences ne sont pas habituelles. Dans ce court texte, nous tentons de jeter des ponts entre ces disciplines. Nous présentons une série de capsules interreliées visant à illustrer à quoi ressemblerait une perspective plus interdisciplinaire (lorsqu'elle porte fruit) ainsi que ses avantages. Concrètement, nous nous penchons sur un sujet récent, notamment les rythmes animaux dans les interactions, que nous étudions au regard de différentes approches. Nous présentons cinq efforts de recherche qui, selon nous, établissent avec succès un lien entre cinq domaines scientifiques particuliers de la recherche sur le rythme, conceptualisés comme suit : les neurosciences sociales, la quantification rythmique détaillée, l'ontogenèse, les approches informatiques, et les interactions spontanées. Nos suggestions inspireront, nous l'espérons, l'apparition d'un champ intitulé « rythmes comparatifs dans le cadre d'interactions », qui pourrait intégrer les connaissances des domaines de la zoologie, de la psychologie comparative, des neurosciences et de l'informatique, et miser sur de telles connaissances.

Mots-clés : rythmes communicatifs dans les comportements, rythmes cérébraux, cognition comparative, ontogenèse du rythme, modèles fondés sur des agents

\section{References}

Aihara, I. (2009). Modeling synchronized calling behavior of Japanese tree frogs. Physical Review E, 80, 011918. http://dx.doi.org/10.1103/ PhysRevE.80.011918

Aihara, I., Mizumoto, T., Otsuka, T., Awano, H., Nagira, K., Okuno, H. G., \& Aihara, K. (2014). Spatio-temporal dynamics in collective frog choruses examined by mathematical modeling and field observations. Scientific Reports, 4, 3891. http://dx.doi.org/10.1038/srep03891

Aihara, I., Takeda, R., Mizumoto, T., Otsuka, T., Takahashi, T., Okuno, H. G., \& Aihara, K. (2011). Complex and transitive synchronization in a frustrated system of calling frogs. Physical Review E, 83, 031913. http://dx.doi.org/10.1103/PhysRevE.83.031913

Aronov, D., Veit, L., Goldberg, J. H., \& Fee, M. S. (2011). Two distinct modes of forebrain circuit dynamics underlie temporal patterning in the vocalizations of young songbirds. The Journal of Neuroscience, 31, 16353-16368. http://dx.doi.org/10.1523/JNEUROSCI.3009-11.2011

Assaneo, M. F., Ripollés, P., Orpella, J., Lin, W. M., de Diego-Balaguer, R., \& Poeppel, D. (2019). Spontaneous synchronization to speech reveals neural mechanisms facilitating language learning. Nature Neuroscience, 22, 627-632. http://dx.doi.org/10.1038/s41593-019-0353-Z

Babiloni, F., \& Astolfi, L. (2014). Social neuroscience and hyperscanning techniques: Past, present and future. Neuroscience and Biobehavioral Reviews, 44, 76-93. http://dx.doi.org/10.1016/j.neubiorev.2012.07.006

Bee, M. A., \& Micheyl, C. (2008). The cocktail party problem: What is it? How can it be solved? And why should animal behaviorists study it? Journal of Comparative Psychology, 122, 235-251. http://dx.doi.org/10 $.1037 / 0735-7036.122 .3 .235$

de Boer, B. (2001). The origins of vowel systems (Vol. 1). New York, NY: Oxford University Press on Demand. 
de Boer, B., \& Kuhl, P. K. (2003). Investigating the role of infant-directed speech with a computer model. Acoustics Research Letters Online, 4, 129-134. http://dx.doi.org/10.1121/1.1613311

Dikker, S., Wan, L., Davidesco, I., Kaggen, L., Oostrik, M., McClintock, J., . . . Poeppel, D. (2017). Brain-to-brain synchrony tracks real-world dynamic group interactions in the classroom. Current Biology, 27, 1375-1380. http://dx.doi.org/10.1016/j.cub.2017.04.002

Hamilton, W. D. (1971). Geometry for the selfish herd. Journal of Theoretical Biology, 31, 295-311. http://dx.doi.org/10.1016/00225193(71)90189-5

Herbers, J. M. (2013). 50 years on: The legacy of William Donald Hamilton. Biology Letters, 9, 20130792. http://dx.doi.org/10.1098/rsbl.2013 .0792

Hopkins, C. D. (1988). Neuroethology of electric communication. Annual Review of Neuroscience, 11, 497-535.

Hyland Bruno, J. (2017). Song rhythm development in zebra finches. New York, NY: City University of New York.

Hyland Bruno, J., \& Tchernichovski, O. (2019). Regularities in zebra finch song beyond the repeated motif. Behavioural Processes, 163, 53-59. http://dx.doi.org/10.1016/j.beproc.2017.11.001

Jürgens, U. (2009). The neural control of vocalization in mammals: A review. Journal of Voice, 23, 1-10. http://dx.doi.org/10.1016/j.jvoice .2007.07.005

Kelley, D. B., \& Bass, A. H. (2010). Neurobiology of vocal communication: Mechanisms for sensorimotor integration and vocal patterning. Current Opinion in Neurobiology, 20, 748-753. http://dx.doi.org/10 .1016/j.conb.2010.08.007

Konishi, M. (1965). The role of auditory feedback in the control of vocalization in the white-crowned sparrow. Zeitschrift für Tierpsychologie, 22, 770-783.

Krakauer, J. W., Ghazanfar, A. A., Gomez-Marin, A., MacIver, M. A., \& Poeppel, D. (2017). Neuroscience needs behavior: Correcting a reductionist bias. Neuron, 93, 480-490. http://dx.doi.org/10.1016/j.neuron .2016 .12 .041

Lindenberger, U., Li, S.-C., Gruber, W., \& Müller, V. (2009). Brains swinging in concert: Cortical phase synchronization while playing guitar. BMC Neuroscience, 10, 22. http://dx.doi.org/10.1186/1471-2202$10-22$

Liu, D., Liu, S., Liu, X., Zhang, C., Li, A., Jin, C., . . Zhang, X. (2018). Interactive brain activity: Review and progress on EEG-based hyperscanning in social interactions. Frontiers in Psychology, 9, 1862. http:// dx.doi.org/10.3389/fpsyg.2018.01862

Marler, P. (1970). Birdsong and speech development: Could there be parallels? American Scientist, 58, 669-673.

Merker, B. H., Madison, G. S., \& Eckerdal, P. (2009). On the role and origin of isochrony in human rhythmic entrainment. Cortex, 45, 4-17. http://dx.doi.org/10.1016/j.cortex.2008.06.011
Mizumoto, T., Aihara, I., Otsuka, T., Takeda, R., Aihara, K., \& Okuno, H. G. (2011). Sound imaging of nocturnal animal calls in their natural habitat. Journal of Comparative Physiology A, 197, 915-921. http://dx .doi.org/10.1007/s00359-011-0652-7

Okobi, D. E., Jr., Banerjee, A., Matheson, A. M. M., Phelps, S. M., \& Long, M. A. (2019). Motor cortical control of vocal interaction in neotropical singing mice. Science, 363, 983-988. http://dx.doi.org/10 $.1126 /$ science.aau9480

Ota, K., Aihara, I., \& Aoyagi, T. (2019). Interaction mechanisms quantified from dynamical features of frog choruses. arXiv preprint arXiv:1907. 11403. Retrieved from https://arxiv.org/abs/1907.11403

Pika, S., Wilkinson, R., Kendrick, K. H., \& Vernes, S. C. (2018). Taking turns: Bridging the gap between human and animal communication Proceedings of the Royal Society B: Biological Sciences, 285, 20180598.

Ravignani, A. (2014). Chronometry for the chorusing herd: Hamilton's legacy on context-dependent acoustic signalling-A comment on Herbers (2013). Biology Letters, 10, 20131018. http://dx.doi.org/10.1098/ rsbl.2013.1018

Ravignani, A. (2019). Timing of antisynchronous calling: A case study in a harbor seal pup (Phoca vitulina), Journal of Comparative Psychology, 133, 272-277.

Ravignani, A., \& de Reus, K. (2019). Modelling animal interactive rhythms in communication. Evolutionary Bioinformatics, 15 , 1176934318823558. http://dx.doi.org/10.1177/1176934318823558

Ravignani, A., Verga, L., \& Greenfield, M. D. (2019). Interactive rhythms across species: The evolutionary biology of animal chorusing and turntaking. Annals of the New York Academy of Sciences, 1453, 12-21. http://dx.doi.org/10.1111/nyas.14230

Schilbach, L., Timmermans, B., Reddy, V., Costall, A., Bente, G., Schlicht, T., \& Vogeley, K. (2013). Toward a second-person neuroscience. Behavioral and Brain Sciences, 36, 393-414. http://dx.doi.org/10.1017/ S0140525X12000660

Schwartz, J. J., \& Freeberg, T. M. (2008). Acoustic interaction in animal groups: Signaling in noisy and social contexts. Journal of Comparative Psychology, 122, 231-234. http://dx.doi.org/10.1037/0735-7036.122.3 .231

Strogatz, S. (2004). Sync: The emerging science of spontaneous order. London, United Kingdom: Penguin.

Toussaint, G. T. (2019). The geometry of musical rhythm: What makes a "good" rhythm good? (2nd ed.). Boca Raton, FL: Chapman and Hall/ CRC. http://dx.doi.org/10.1201/9781351247771

Received October 17, 2019 Accepted May 12, 2020 